\title{
INFLUENCIA DE UN PROGRAMA PARA EL DESARROLLO DEL APRENDIZAJE EXPERIENCIAL EN AGRICULTORES
}

\section{Influence of a program for the development of experiential learning in farmers}

Henry Alarcón-Diaz*, Noel Alcas Zapata**, Mitchell Alarcón Díaz***, Yolvi Ocaña Fernández****, Ronald M Hernández*****, Antonio Rodríguez Fuentes $* * * * * *$

\section{Resumen}

El objetivo del presente estudio ha sido llevar a la práctica el programa andragógico "Cultivos Orgánicos” para el desarrollo del aprendizaje experiencial. En esta investigación participaron un grupo de 60 agricultores de una comunidad ubicada al sur de Lima (Perú). El método empleado fue mixto y se desarrolló en dos momentos: El primero, con un enfoque cuantitativo $y$ un diseño cuasi experimental se recabaron datos que fueron sometidos a un pretest y postest a través del software SPSS, y el segundo, con un enfoque cualitativo se aplicaron entrevistas a profundidad cuyos resultados fueron analizados mediante el software Atlas. Ti. Los resultados cuantitativos obtenidos mostraron una influencia del programa en el aprendizaje experiencial de los pobladores. Por su parte, los resultados cualitativos a partir de las entrevistas, muestran un reconocimiento, valía y aporte del programa según la visión de los entrevistados.

Palabras clave: Experimento educacional. Aprendizaje a través de la experiencia. Formación dirigida a adultos. Diversidad cultural. Programa de desarrollo..

\begin{abstract}
The objective of the present study has been to carry out the andragogic program "Organic Crops" for the development of experiential learning. In this research, a group of 60 farmers from a community located south of Lima (Peru) participated. The method used was mixed and was developed in two moments: The first, with a quantitative approach and a quasi-experimental design, data were collected that were submitted to a pretest and post-test through the SPSS software, and the second, with a qualitative approach. They conducted in-depth interviews whose results were analyzed using the Atlas software. You. The quantitative results obtained showed an influence of the program on the experiential learning of the inhabitants. On the other hand, the qualitative results from the interviews show a recognition, value and contribution of the program according to the vision of the interviewees.
\end{abstract}

Keywords: Educational experiment. Learning through experience. Adult-oriented training. Cultural diversity. Development program.

\footnotetext{
* Educador. Docente de la Universidad Nacional de Educación. https://orcid.org/0000-0003-1588-4390

** Educador. Docente de la Universidad César Vallejo. https://orcid.org/0000-0001-9308-4319

*** Educador. Docente de la Universidad César Vallejo. https://orcid.org/0000-0003-0027-5701

**** Educador. Docente de la Universidad César Vallejo y de la Universidad Nacional Mayor de San Marcos. https://orcid.org/0000-0002-2566-6875

***** Psicólogo. Coordinador de Producción Científica y Capacitación de la Universidad San Ignacio de Loyola.rhernandezv@usil.edu.pe.https://orcid.org/0000-0003-1263-2454

****** Educador. Docente de la Universidad de Granada. https://orcid.org/0000-0002-8036-9902
} 


\section{INTRODUCCIÓN}

Según Ocampo (2008) la andragogía es una propuesta contemporánea la cual hace posible la aplicación de estrategias, modelos, programas, etc., en contextos socioculturales emergentes. En este espacio dialógico, se hace necesario que se investigue científica y pedagógicamente para creativizar los modelos de enseñanza aprendizaje y generar así cambios en los sujetos participantes (Freire, 1985).

En el Perú, la propuesta peruana dirigida a la escuela rural y especialmente la andragogía está por verse; los programas se formulan de manera sesgada $\mathrm{y}$, en algunos casos, dificultan la aplicación práctica y significativa de lo aprendido por parte de los estudiantes. Teniendo en la ruralidad una posibilidad extensa y valiosa para enriquecer las prácticas cotidianas, urge aquí un tanto aclarar el panorama y establecer normas técnicas que definan adónde se quiere llegar (Tarea, 2017).

Así, hablar de un aprendizaje en una escuela rural y de carácter andragógico es reconocer los espacios geográficos y la cosmovisión de los habitantes y, desde ahí promover saberes para la cristalización de un proyecto de vida. Es reconocer un patrimonio, un estilo único, diferente a la mirada tradicional debido a la interacción entre sujetos, comunidad y naturaleza. Este respeto por la idiosincrasia del poblador es importante ya que genera un espacio de confianza y diálogo (Naranjo y Carrero, 2017; Villadiego, Huffman, Guerrero y Cortecero, 2017; Fernández, 2017; Rodríguez, 2017; Rodríguez y Fernández, 2018). La existencia de comunidades rurales ajenas, en parte, a los estereotipos que propone la mentalidad occidental, posibilita el accionar creativo y distante de las políticas públicas creando posibilidades de autosostenimiento y fortaleciendo objetivos a corto y mediano plazo.

En ese sentido, Ávila (2017) sostiene que:

Encontramos investigaciones que se han realizado sobre experiencias pedagógicas significativas de educación rural, en las cuales se nota una constante preocupación por el mejoramiento de la calidad de la educación, para lo cual proponen: adecuar los contenidos, los métodos de enseñanza y la adaptación curricular, las metodologías, el cronograma de actividades académicas para que no haya interferencia entre las labores académica con las labores del campo (p.127)

Para Azofeifa (2016) el aprendizaje transcultural, de generación en generación, se convierte en uno de los pilares de la educación rural andragógica, el diseñar, por lo tanto, un modelo de aprendizaje rígido, vertical, centrado en el saber del maestro desestima, de las personas, un enorme potencial adquirido a partir de lo aprendido en el día a día. De esta manera, el reto de una propuesta educativa oficial mimetizaría y consideraría un modelo basado en los saberes ancestrales de la comunidad en beneficio de uno actual y vanguardista (Gallego y Rodríguez, 2014; Rodríguez, 2018). Por lo tanto, asumimos que el éxito de un aprendizaje andragógico recae en el autoprendizaje con visión prospectiva ya que bajo este modelo el adulto decide que es lo que desea aprender, en ese sentido, la andragogía respeta la horizontalidad (compartir los logros y compromisos) y la participación (tomar decisiones, compartir y trazar metas). Además, conociendo los intereses del estudiante adulto se podría trazar la metodología a seguir para finalmente ejecutar y posibilitar así la ejecución de actividades productivas (Morales y Leguizamón, 2017; Herrera y Buitrago, 2015).

Dentro de esta complejidad ¿cómo se podría formular una propuesta de aprendizaje para los agricultores?, ¿qué características podría tener este programa? Estas preguntas orientadoras se formularon con el propósito de poner en práctica un programa de cultivo para fortalecer el aprendizaje experiencial de los estudiantes adultos de una comunidad ubicada al sur de Lima.

\section{METODOLOGÍA}

Los participantes forman parte de los pequeños agricultores de una comunidad rural, los mismos que participaron en el Programa sobre "Cultivos Orgánicos", con el propósito de mejorar sus aprendizajes experienciales, dándoles un valor agregado y consistente en incorporar estrategias para este tipo de cultivos. Para el análisis del aprendizaje experiencial se consideró incluir: la experiencia concreta (EC), la observación reflexiva (OR), la 
conceptualización abstracta (CA), y finalmente la experimentación activa (EA). La medición se realizó mediante la aplicación de un instrumento con 30 preguntas que corresponden a cada uno de los cuatro aspectos mencionados líneas arriba.

El enfoque de este estudio fue mixto, según aconseja la metodología de investigación más vanguardista (Cameron, 2011; Díaz, 2014); en un primer momento, con diseño cuasi experimental y con la participación de una muestra de 60 agricultores, de los cuales 30 de ellos formaron el grupo de control y 30 el grupo experimental; la aplicación del programa tuvo una duración aproximada de 4 meses, para lo cual se tomó el pretest antes de iniciar el programa "cultivos orgánicos" y el postest al final del proceso. Para la contrastación de las hipótesis se utilizó el Test U de Mann-Whitney, el cual determinó la influencia del programa "cultivos orgánicos" en el aprendizaje experiencial de los pequeños agricultores. En un segundo momento; con entrevistas a profundidad a 17 sujetos que participaron como estudiantes en el programa, se realizaron las mismas, las cuales fueron analizadas mediante el programa Atlas.ti.

\section{RESULTADOS}

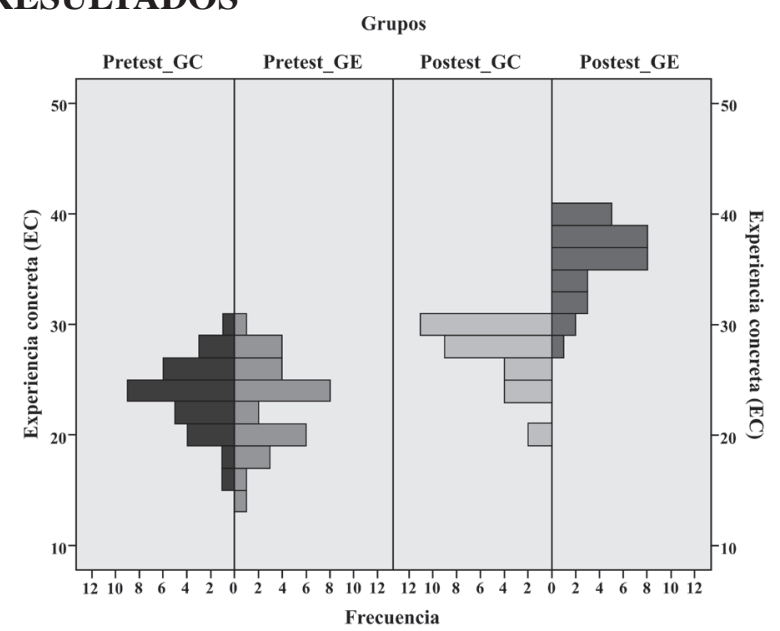

Figura 1. Diagrama pirámide de la experiencia concreta

Con relación a los resultados cuantitativos descriptivos, en la figura 1, se muestra la experiencia concreta por parte de los agricultores. En el diagrama de pirámide, se evidencian las frecuencias de los test del grupo control y experimental. Asimismo, se puede observar que las frecuencias en el pretest de ambos grupos se encuentran en un nivel similar no apreciándose diferencia entre ellos, sin embargo, las frecuencias en el postest del grupo experimental se muestran por encima del postest del grupo control y del pretest del mismo grupo experimental, lo cual indica que la puntuación final lograda en el grupo experimental se debe al proceso de experimentación.

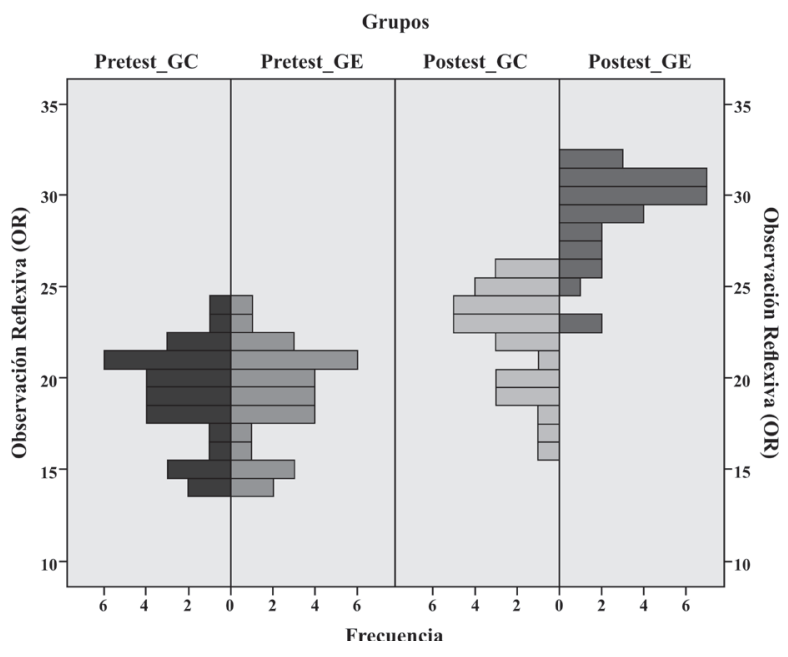

Figura 2. Diagrama pirámide de la observación reflexiva

En la figura 2 se observan los resultados sobre la observación reflexiva. En el diagrama de pirámide, se muestra, también, las frecuencias de los test del grupo control y experimental. Asimismo, se evidencia que las frecuencias en el pretest de ambos grupos se encuentran en un nivel similar no apreciándose diferencia entre ellos, sin embargo, las frecuencias en el postest del grupo experimental se centran por encima del postest del grupo control, lo cual permite afirmar que la puntuación lograda en el grupo experimental se debe al proceso de experimentación.

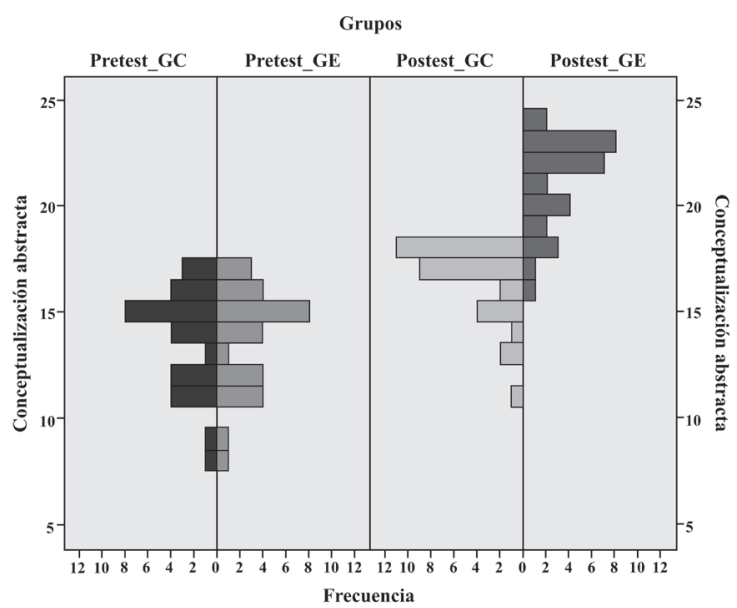

Figura 3. Diagrama pirámide de la conceptualización abstracta 
La figura 3 muestra los resultados sobre la conceptualización abstracta. En tal diagrama de pirámide se observa, además, las frecuencias de los test del grupo control y experimental. Asimismo, se evidencia que las frecuencias en el pretest de ambos grupos se encuentran en un nivel similar no apreciándose diferencia entre ellos, sin embargo, las frecuencias en el postest del grupo experimental se concentran por encima del postest del grupo control, lo cual permite afirmar que la puntuación lograda en el grupo experimental se debe al proceso de experimentación.

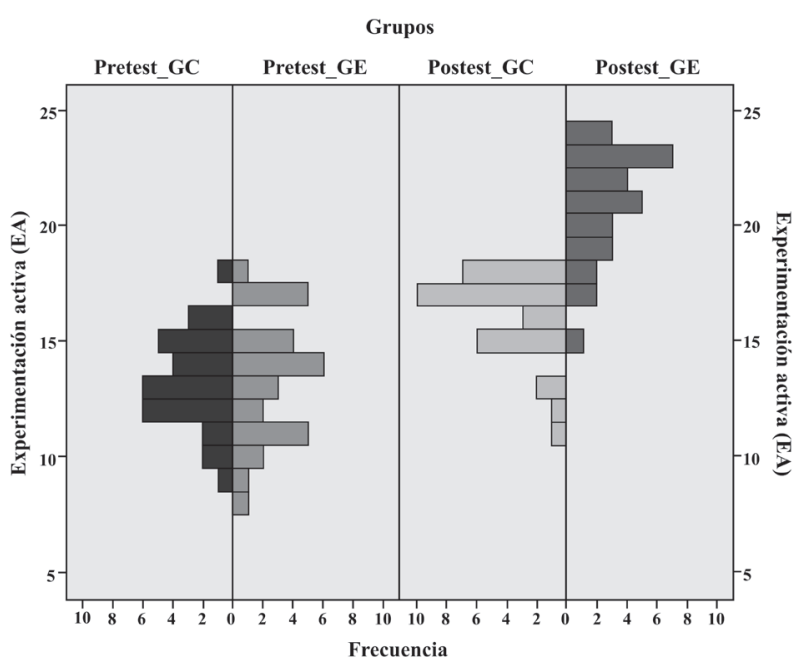

Figura 4. Diagrama pirámide de la experimentación activa

La figura 4 contiene los resultados sobre la experimentación activa de los agricultores. En el diagrama de pirámide, se observa también, la comparación de los test del grupo control y experimental en referencia al pretest y postest. Asimismo, se evidencia que las frecuencias en el pretest de ambos grupos se encuentran en un nivel similar, no apreciándose diferencia entre ellos, sin embargo, las frecuencias en el postest del grupo experimental se centran por encima del postest del grupo control, lo cual permite afirmar que la puntuación lograda en el grupo experimental se debe al proceso de experimentación.

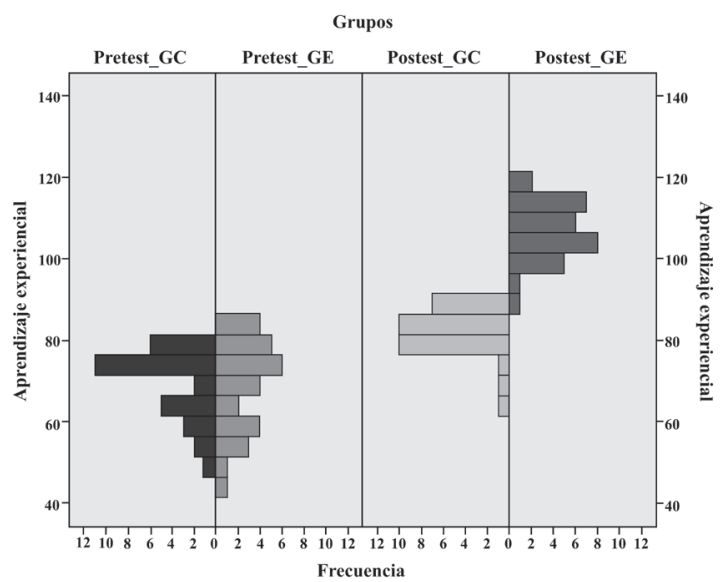

Figura 5. Diagrama pirámide del aprendizaje experiencial

En la figura 5 se presentan los resultados sobre el aprendizaje experiencial de los agricultores. Se observa, además, la comparación de los test del grupo control y experimental en cuanto al pre y postest. Asimismo, se evidencia que las comparaciones en el pretest de ambos grupos se encuentran en un nivel similar, no apreciándose diferencia entre ellos, sin embargo, las frecuencias en el postest del grupo experimental se entran por encima del postest del grupo control, lo cual permite afirmar que la puntuación lograda en el grupo experimental se debe al proceso de experimentación.

Tabla 1.

Nivel de significación del aprendizaje experiencial de pequeños agricultores

\begin{tabular}{lcccc}
\hline EST & N & $\begin{array}{c}\text { Rango } \\
\text { promedio }\end{array}$ & $\begin{array}{c}\text { Suma } \\
\text { de rangos }\end{array}$ & $\begin{array}{c}\text { Test U de } \\
\text { Mann-Whitney }\end{array}$ \\
\hline $\begin{array}{l}\text { Postest_Grupo } \\
\text { de control }\end{array}$ & 30 & 15,62 & 468,50 & $\mathrm{U}=3,500$ \\
$\begin{array}{l}\text { Postest_Grupo } \\
\text { experimental }\end{array}$ & 30 & 45,38 & 1361,50 & $\mathrm{Z}=-6,605$ \\
& 60 & & Sig. asintót $=0.000$
\end{tabular}

Fuente: Resultados obtenidos en base a SPSS. Significancia estadística $* \mathrm{p}<1 \%, \mathrm{p}^{* *}<5 \%$.

Con relación a los resultados cuantitativos inferenciales, en la tabla 1 se muestran los resultados del postest del grupo de control y postest del grupo experimental, donde el nivel de $\mathrm{z}$ se encuentra por debajo del nivel crítico zc $=-6.605$ y $p_{-}$valor $=0.000$ $<0,05$, lo cual implica rechazar H0. Por lo tanto, la aplicación del Programa "cultivos orgánicos" influye en aprendizaje experiencial de pequeños agricultores. 
Tabla 2.

Nivel de significación de la experiencia concreta de los pequeños agricultores

\begin{tabular}{lcccc}
\hline EST & N & $\begin{array}{c}\text { Rango } \\
\text { promedio }\end{array}$ & $\begin{array}{c}\text { Suma } \\
\text { de rangos }\end{array}$ & $\begin{array}{c}\text { Test U de } \\
\text { Mann-Whitney }\end{array}$ \\
\hline $\begin{array}{l}\text { Postest_Grupo } \\
\text { de control }\end{array}$ & 30 & 16,90 & 507,00 & $\mathrm{U}=42,000$ \\
$\begin{array}{l}\begin{array}{l}\text { Postest_Grupo } \\
\text { experimental }\end{array} \\
30\end{array}$ & 44,10 & 1323,00 & $\mathrm{Z}=-6,869$ \\
& 60 & & Sig. asintót $=0.000$ \\
\hline
\end{tabular}

Fuente: Resultados obtenidos en base a SPSS. Significancia estadística $* \mathrm{p}<1 \%, \mathrm{p} * *<5 \%$.

En la tabla 2, se muestran los resultados del postest del grupo de control y postest del grupo experimental, donde el nivel de $\mathrm{z}$ se encuentra por debajo del nivel crítico $\mathrm{zc}=-6.869$ y $\mathrm{p}_{-}$valor $=0.000<0,05$, lo cual implica rechazar H0. Por lo tanto, la aplicación del Programa "Cultivos orgánicos" influye en la experiencia concreta de los pequeños agricultores.

Tabla 3.

Nivel de significación de la observación reflexiva de los pequeños agricultores

\begin{tabular}{|c|c|c|c|c|}
\hline EST & $\mathrm{N}$ & $\begin{array}{l}\text { Rango } \\
\text { promedio }\end{array}$ & $\begin{array}{c}\text { Suma } \\
\text { de rangos }\end{array}$ & $\begin{array}{c}\text { Test U de } \\
\text { Mann-Whitneya }\end{array}$ \\
\hline $\begin{array}{l}\text { Postest_Grupo } \\
\text { de control }\end{array}$ & 30 & 16,73 & 502,00 & $\mathrm{U}=37,000$ \\
\hline $\begin{array}{l}\text { Postest_Grupo } \\
\text { experimental }\end{array}$ & 30 & 44,27 & 1328,00 & $Z=-6,128$ \\
\hline 60 & & & \multicolumn{2}{|c|}{ Sig. asintót $=0.000$} \\
\hline
\end{tabular}

Fuente: Resultados obtenidos en base a SPSS. Significancia estadística $* \mathrm{p}<1 \%, \mathrm{p} * *<5 \%$.

En la tabla 3, se muestran los resultados del postest del grupo de control y postest del grupo experimental, donde el nivel de $\mathrm{z}$ se encuentra por debajo del nivel crítico $\mathrm{zc}=-6.128 \mathrm{y} \mathrm{p}$ _ valor $=0.000<0,05$, lo cual implica rechazar H0. Por lo tanto, la aplicación del Programa "cultivos orgánicos" influye en la observación reflexiva de los pequeños agricultores.
Tabla 4.

Nivel de significación de la conceptualización abstracta de los pequeños agricultores

\begin{tabular}{lcccc}
\hline EST & N & $\begin{array}{c}\text { Rango } \\
\text { promedio }\end{array}$ & $\begin{array}{c}\text { Suma } \\
\text { de rangos }\end{array}$ & $\begin{array}{c}\text { Test U de } \\
\text { Mann-Whitneya }\end{array}$ \\
\hline $\begin{array}{l}\text { Postest_Grupo } \\
\text { de control }\end{array}$ & 30 & 17,27 & 518,00 & $\mathrm{U}=53,000$ \\
$\begin{array}{l}\text { Postest_Grupo } \\
\text { experimental }\end{array}$ & 30 & 43,73 & 1312,00 & $\mathrm{Z}=-5,935$ \\
& 60 & & Sig. asintót $=0.000$ \\
\hline
\end{tabular}

Fuente: Resultados obtenidos en base a SPSS. Significancia estadística $* \mathrm{p}<1 \%, \mathrm{p}^{* *}<5 \%$.

La tabla 4 muestra los resultados del postest del grupo de controly postest del grupo experimental, donde el nivel de z se encuentra por debajo del nivel crítico $\mathrm{zc}=-5.935 \mathrm{y} \mathrm{p}_{-}$valor $=0.000<0,05$, lo cual implica rechazar H0. Por lo tanto, la aplicación del Programa "cultivos orgánicos" influye en la conceptualización abstracta de los pequeños agricultores.

Tabla 5.

Nivel de significación de la experimentación activa de los pequeños agricultores

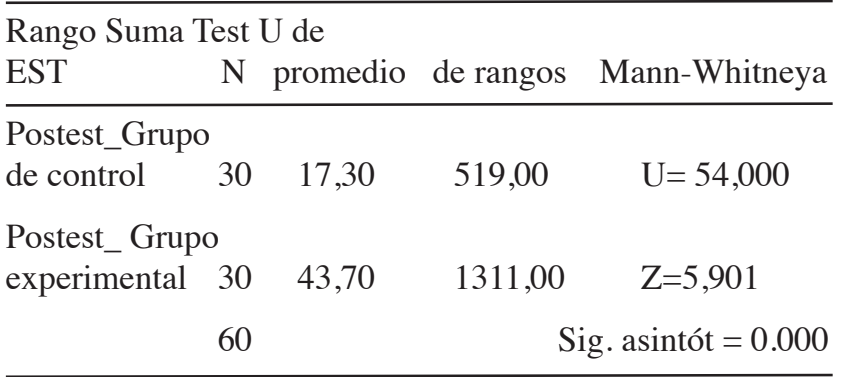

Fuente: Resultados obtenidos en base a SPSS. Significancia estadística $* \mathrm{p}<1 \%, \mathrm{p} * *<5 \%$.

La tabla 5 contiene los resultados del postest del grupo de control y postest del grupo experimental, donde el nivel de $\mathrm{z}$ se encuentra por debajo del nivel crítico zc $=-5.901$ y $p_{-}$valor $=0.000<0,05$, lo cual implica rechazar H0. Por consiguiente, La aplicación del Programa "Cultivos orgánicos" influye en la experimentación activa de los pequeños agricultores. 
Con el propósito de profundizar y llegar a un entendimiento mayor de los resultados cuantitativos, se realizó un análisis cualitativo el mismo que esta ordenado por categorías, códigos y la interpretación respectiva (Tabla 6).

Tabla 6.

Aspectos vinculados al Programa "Cultivos orgánicos"

\begin{tabular}{ll}
\hline Categoría & \multicolumn{1}{c}{ Códigos } \\
\hline $\begin{array}{l}\text { Programa } \\
\text { "Cultivos orgánicos" }\end{array}$ & $\begin{array}{l}\text { 1. Actividades interesante } \\
\text { 2. Importante uso de recursos } \\
\text { 3. Variedad del programa } \\
\text { 4. Aprendizaje de los temas }\end{array}$ \\
\hline
\end{tabular}

Fuente: Resultados consolidados y obtenidos en base a Atlas.ti.

A partir de lo expuesto por los estudiantes agricultores, el programa incluye una serie de actividades las cuales resultan motivadoras. El empleo de recursos propios del lugar que se vinculan de manera directa con el día a día del agricultor se resalta. Otro aspecto relevante son los contenidos incluidos en la experimentación ya que se prevalece el aprender haciendo e intercambiando actividades de manera seguida; finalmente se destaca el aprendizaje a partir de objetivos claramente definidos al inicio de la aplicación de la intervención en el grupo de sujetos.

Tabla 7.

Aspectos vinculados a la enseñanza y aprendizaje

\begin{tabular}{lll}
\hline Categorías & \multicolumn{2}{c}{ Códigos } \\
\hline Materiales empleados & $\begin{array}{l}\text { 1. Fácil uso de las guías de trabajo } \\
\text { 2. Aplicación de fichas en los } \\
\text { talleres }\end{array}$ \\
Seguimiento al docente & $\begin{array}{l}\text { 3. Aplicación constante del } \\
\text { profesor en clase } \\
\text { 4. Evaluaciones constantes }\end{array}$
\end{tabular}

Fuente: Resultados consolidados y obtenidos en base a Atlas.ti.
De acuerdo a lo dicho por los pequeños agricultores, la enseñanza y aprendizaje vivenciado destaca los materiales los cuales no son de uso complejo. Aquí se emplearon guías las cuales fueron discutidas para luego aplicarlas en el campo mismo. Por otro lado, la labor del docente se ve reflejada en el acompañamiento constante durante las sesiones de clase, despejando dudas, sugiriendo actividades y evaluando de manera seguida los contenidos del programa "cultivos orgánicos".

\section{DISCUSIÓN}

A decir de D’Amico y De Benedictis (2018) un modelo andragógico se orienta a asumir cambios, incentivar el juicio crítico y en la posibilidad de tomar decisiones por parte de los adultos participantes.

Así, la aplicación del programa "cultivos orgánicos" contribuyó a valorizar los saberes de los agricultores y a tener un punto de inicio para emprender una forma de auto sostenimiento basado en el cultivo. El programa aplicado influyó en los participantes a partir de la mejora en la experiencia concreta, la observación reflexiva, la conceptualización abstracta, y finalmente la experimentación activa. También durante el proceso, se tomaron en cuenta aquellos conocimientos los cuales han sido acumulados a través de la vida misma y las que toman, a partir de la teoría, valor al momento de decidir en determinadas situaciones (Vallejo, 2016).

El aplicar intervenciones en el ámbito rural y que estas tengan una simetría con la idiosincrasia de los pobladores es un factor determinante para el éxito; propuestas muy alejadas del contexto fracasaron por intentar imponer actitudes o costumbres diferentes a las que están arraigadas a través del tiempo y en la gente misma. Aquí se resalta la idea de que no basta con conocer contenidos ni metodologías (Rumbo, 2016).

Al finalizar el programa "cultivos orgánicos" se destacó la labor de los docentes a cargo ya que ellos motivaron el aprendizaje experiencial de los agricultores. Uno de los aspectos que se tuvo en cuenta fue el identificar las características y necesidades de los sujetos participantes; de esta manera y a la par con 
estudios similares, se propuso un diseño instruccional a partir de lo que los participantes desean y necesitan (Rodríguez y Gómez, 2018).

Es recomendable continuar esta indagación de comprobación de la eficacia y eficiencia del aprendizaje experiencial en este y otros colectivos, dada la pertinencia de los resultados y la necesidad latente de sus aprendizajes. Se anima, por tanto, a otros a investigadores a perpetuar esta necesidad y modelo de aprendizaje, como se ha venido haciendo hasta el momento (Jarin, 2005; Castro, 2010).

\section{REFERENCIAS}

Ainnsh, R. (2016). Thoughtfully Designed Online Courses as Effective Adult Learning Tools. Journal of Adult Education, 45(1), 7-9. Recuperado de http://0-search.proquest.com.millenium.itesm. $\mathrm{mx} /$ docview/1786240807?accountid=41938

Ávila, B. (2017). Experiencias pedagógicas significativas de educación rural en Colombia, Brasil y México. Revista del Centro de Investigación. Universidad La Salle, 14 (48), 121-158. DOI: http://dx.doi.org/10.26457/recein. v14i48.1486

Azofeifa, J. (2016). Evolución conceptual e importancia de la andragogía para la optimización del alcance de los programas y proyectos académicos universitarios de desarrollo rural. Revista Educare, 21(1), pp. 1-16. DOI: http:// dx.doi.org/10.15359/ree.21-1.23

Cameron, R. (2011). Mixed Methods Research: The five Ps framework. The Electronic Journal of Business Research Methods, 9 (2), 96-108. 2011. Recuperado de http://www.ejbrm.com.

Castro, M. (2010). El nivel de conocimiento de andragogía y el desarrollo de la especialidad de educación básica. Tesis de Doctorado. Universidad Nacional de Educación Enrique Guzmán y Valle.

D’Amico-López, R. y De Benedictis, G. (2018). Inclusión de la andragogía como materia en el currículo de la carrera de medicina. FEM: Revista de la Fundación Educación Médica,
21(3), 165. Recuperado de http://scielo. isciii.es/scielo.php?script=sci_arttext\&pid $=$ S2014-98322018000300009

Díaz, S. M. (2014), Los métodos mixtos de investigación: presupuestos generales y aporte a la evaluación educativa. Revista Portuguesa de Pedagogía, 48 (1), 7-23. 2014. DOI: 10.14195/1647 8614_48 1_1

Fernández, A. (2017). Culturas en el aula vistas por el alumnado. Actitudes de alumnos de cultura mayoritaria ante compañeros de minoritarias. Revista RETOS XXI, 1(1), 16-35. Recuperado a partir de http://revistas.utp.ac.pa/index.php/ retoxxi/article/view/1517

Freire,P.(1985).Pedagogía del oprimido. Montevideo: Tierra Nueva.

Galbraith, D. D. \& Fouch, S. E (2007). Principles of Adult Learning: Application to Safety Training. Professional Safety, 52(9), 35-40. Recuperado de http://0-search.proquest.com.millenium.itesm. $\mathrm{mx} /$ docview $/ 200415451$ ? accountid $=41938$

Gallego, J.L. y Rodríguez, A. (2014). El reto de una educación de calidad en la Escuela Inclusiva. Revista Portuguesa de Pedagogía, 48(1), 1-24. Recuperado de http://impactum-journals.uc.pt/ index.php/rppedagogia/article/view/2237/1476

Herrera, L. y Buitrago, R. (2015). Educación rural en Boyacá, fortalezas y debilidades desde la perspectiva del profesorado. Praxis \& Saber, 12 (6), pp.169-190. Recuperado de https://revistas. uptc.edu.co/index.php/praxis_saber/article/ view/3768/3550

Jarrin, C. (2005). Metodología andragógica aplicada en el proceso de aprendizaje del 2 do curso complementario de licenciatura en Enfermería y propuesta de guía metodológica. Universidad Central del Ecuador. Tesis para optar el Grado de Magister.

Kaufman, E. (2015). Correlation Study of Adult Educators'Facilitation Experience, professional/ Academic Discipline, and Andragogy Practices (Order No. 3716664). Available from ProQuest Dissertations \& Theses Global. Recuperado de http://0-search.pro-quest.com.millenium.itesm. $\mathrm{mx} /$ docview $/ 1711165909$ ?accountid $=41938$ 
Knowles, M. S., Holton, E. F., \& y Swanson, R. A. (2005). The Adult Learner: The Definitive Classic in Adult Education and Human Resource Development. Routledge.

Morales, O. y Leguizamón, M. (2017). Teoría andragógica: aciertos y desaciertos en la formación docente en tic. Praxis \& Saber, 19(9). pp. 161181. DOI: https://doi.org/10.19053/22160159. v9.n19.2018.7926

Naranjo, D. y Carrero, A. (2017). Retos y desafíos de la Educación rural para niños y jóvenes en escenarios de Construcción de Paz: una mirada desde lo local para la transformación global Prospectiva. Revista de Trabajo Social e intervención social, 24, pp. 95-120. DOI: https://doi.org/10.25100/ prts.v\%vi\%i.4546

Ocampo, J. (2008). Paulo Freire y la pedagogía del oprimido. Rhela, 10, pp.57-72. Recuperado de https://www.redalyc.org/articulo.oa? id=869 01005

Rodríguez, A. (2017). Profesorado, progenitores y alumnado ante la diversidad sociocultural. Confluencia de perfiles e influencia entre ellos. Teoría de la Educación. Revista Interuniversitaria, 29 (2), 211-231. DOI: http://dx.doi.org/10.14201/ teoredu292211231

Rodríguez, A. (2018). Editorial. Expansión postmoderna tecnológica, escuela inclusiva tecnológica. RETOS XXI, 2, 6-12. DOI: https:// doi.org/10.33412/retoxxi.v2.1.2055

Rodríguez, A. y Fernández, A. D. (2018). Actitudes ante la diversidad cultural de progenitores y descendientes. Eficiencia de la influencia por su grado de confluencia. Arbor, 194 (788), 1-15. DOI: https://doi.org/10.3989/arbor.2018.788n2011

Rodríguez, L. y Gómez, M. (2018). Calidad andragógica en un curso de capacitación de seguridad en el trabajo basado en tecnología.
Revista de Educación y Desarrollo, 44, pp. 49-59. Recuperado de http://www.cucs.udg.mx/revistas/ edu_desarrollo/anteriores/44/44_Rodriguez.pdf.

Rumbo, B. (2016). Problemas y retos de la educación de las personas adultas. Revista Educar, 52 (1), pp. 93-106. DOI: http://dx.doi.org/10.5565/rev/ educar.707

Sánchez, S. (2013). Diagnóstico de las necesidades de capacitación andragógicas de los docentes del quinto año de la Escuela de Derecho de la Universidad Técnica de Machala, para optimizar su labor profesional, Diseño de un Modo Alternativo. Tesis para grado de Magister. Universidad de Guayaquil.

Tarea (2017). Revista de educación y cultura. Lima: Tarea

Taylor, B. \& Kroth, M. (2009). Andragogy's Transition into the Future: Meta-Analysis of Andragogy and Its Search for a Measurable Instrument. Journal of Adult Education, 38(1), 1-11. Recuperado de http://files.eric.ed.gov/fulltext/EJ891073.pdf

Torres, M., Fermín, Y., Arroyo, C. \& Piñero M. (2000). La horizontalidad y la participación en la andragogía. Universidad de los Andes. Recuperado de http://www.saber.ula.ve/ bitstream/123456789/19444/1/articulo4-10-3 . pdf.

Vallejo, L. (2016). Características de los programas de capacitación andragógicos y los procesos de aprendizaje la seguridad minera peruana. RIIGEO, 19 (37), pp. 111-116. Recuperado de http://revistasinvestigacion.unmsm.edu.pe/index. php/iigeo/article/view/12962/11578

Villadiego, J., Huffman, D., Guerrero, S., \& Cortecero, A. (2017). Base pedagógica para generar un modelo no formal de educación ambiental. Luna Azul. 44, pp. 316-333. DOI: https://doi. org/10.17151/luaz.2017.44.19. 